\title{
A Major Optical Flare on the Recently Discovered X-ray Active dMe Star G102-21
}

\author{
I. Pagano ${ }^{1}$, R. Ventura ${ }^{1}$, M. Rodono $\dot{~}^{1}$, G. Peres ${ }^{2}$, G. Micela $^{2}$ \\ 1 Osservatorio Astrofisico di Catania and Istituto di Astronomia dell'Università degli \\ Studi, viale A. Doria 6, I-95125 Catania, Italy \\ 2 Istituto e Osservatorio Astronomico di Palermo, Palazzo dei Normanni, \\ I-90134 Palermo, Italy
}

During $U B V$ photoelectric measurements of G102-21 - a dM3 star recently discovered as a remarkably active X-ray source by Micela et al. (1995) - we have observed the flare shown in Fig. 1 (left panel). The observed flux increase at flare maximum and the energy output (cf. Table 1) make this event one of the largest amplitude and most energetic flares ever detected on UV Cet-type stars.

The ratios $\mathrm{E}_{U} / \mathrm{E}_{B}(=8.9)$ and $\mathrm{E}_{U} / \mathrm{E}_{V}(=3.9)$ are more than 7 and 2 times larger, respectively, than the mean values found for typical dMe flares by Lacy et al. (1976). Moreover, assuming the empirical relation between the absolute magnitude $\mathrm{M}_{V}$ and the mean energy dissipation during a flare (Gurzadyan 1980), the energy released in the $U$ band exceeds the predicted one by a factor of $\sim 3 \cdot 10^{3}$. Clearly, we observed a rather peculiar and rare event that does not appear to be a typical flare on red-dwarf stars.

An aspect that deserves attention is the apparently abrupt descent to the quiescent level $\left(\sim 0^{\mathrm{m}} 7 \mathrm{~min}^{-1}\right)$. Only very few similar cases have been observed (Henry \& Hall 1991, Cristaldi \& Rodonò 1970, Foing et al. 1994). A possible interpretation is that we observed only the upper flaring region of a flare occuring close to or behind the stellar limb, so that the energy emission from low lying and densest flare sites, where the slow decay is produced, was not observed. Otherwise, the flare might have occurred just at or behind the star limb, so that, during the descent phase, the low-lying flare site eventually disappeared behind the limb due to the rotation of the star or was invisible all through the flare duration, respectively.

If the energy released during the course of the flare had been previously stored in a filament, as for solar two-ribbon flares, then a surface magnetic field of few thousands Gauss for a loop semi-length of $1 R_{*}$, and a binary separation of $3-16 R_{\odot}$, are implied (see Pagano et al. 1995 for details).

Table 1. Characteristics of the flare shown in Fig. 1

\begin{tabular}{lccc}
\hline & $U$ & $B$ & $V$ \\
\hline$\Delta m \max (\mathrm{mag})$ & 7.29 & 3.88 & 3.03 \\
Flare energy $^{a}\left(\times 10^{34} \mathrm{erg}\right)$ & $8.9 \pm 3.0$ & $1.0 \pm 0.3$ & $2.3 \pm 0.8$ \\
Equivalent duration $^{\mathrm{a}}$ (hours) & 79 & 3.4 & 1.5 \\
\hline${ }^{a}$ assuming $\mathrm{d}=21.3 \pm 3.6 \mathrm{pc}$ (Gliese \& Jahreiss & $1991)$
\end{tabular}



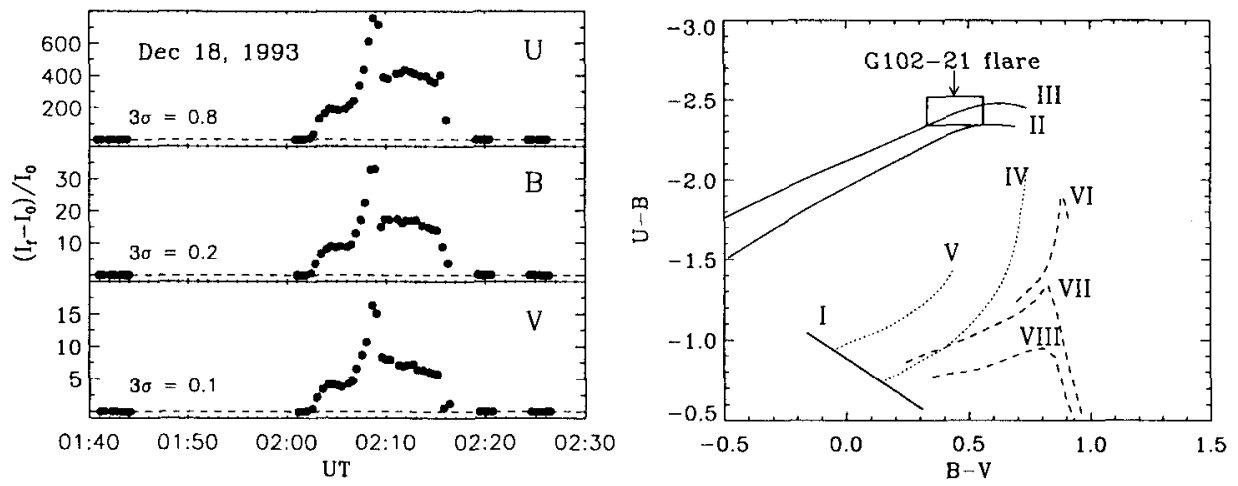

Fig. 1. Left panel: the light curves of the G102-21 flare. Note the quite different ordinate scale on the left subpanels. Right panel: two colour diagram for the flare energy emission. The labelled lines correspond to the following models for flare emission, (I) blackbody at 8,000-20,000 K; (II-III) hydrogen plasmas, optically thin in the Balmer continuum, with $T_{e}=10,000 \mathrm{~K}$ and electron density $10^{12}$ and $10^{14} \mathrm{~cm}^{-3}$, respectively; (IV-V) an optically thick plasma at $\mathrm{T}_{e}=10,000$ and $15,000 \mathrm{~K}$, respectively; (VI-VII-VIII) upper layers of a dwarf star heated by proton beams with threshold proton energy 1 , 2 and $5 \mathrm{MeV}$, respectively. The theoretical model grid is from Gershberg et al. (1991).

The rectangle in Fig. 1 (right panel) delimits the range of variability of $U-B$ and $B-V$ colours for the pure flare radiation during the event. The $U-B$ $(-2.43 \pm 0.09)$ is extremely negative when compared to typical $U-B$ flare colours on dMe stars, usually in the range from -0.5 to -1.5 (Gurzadyan 1980, Cristaldi \& Rodonò 1975). Instead, the $B-V$ colour (0.45 \pm 0.09$)$ is quite typical for stellar flares. In the same Fig. 1, theoretical models from Gershberg et al. (1991) are also shown. The colours of the pure flare radiation fit quite well with the colours predicted by Minikulov (1987) for a hydrogen plasma at $10,000 \mathrm{~K}$, optically thin in the Balmer continuum, with electron density of $10^{14} \mathrm{~cm}^{-3}$.

Acknowledgements: Stellar activity research at Catania Observatory and University is funded by the Italian MURST-CRA, CNR-GNA and ASI.

\section{References}

Cristaldi S., Rodonò M., 1970, A\&AS 2, 223

Cristaldi S., Rodonò M., 1975, in IAU Symp. 67, 75

Foing B.H. et al., 1994, A\&A, 292, 543

Gershberg R.E. et al., 1991, SvA 35, 269

Gliese W., Jahreiss H., 1991, 3th Catalogue of Nearby Stars, ADC, GSFC

Gurzadyan G.A., 1980, Flare Stars, Pergamon, Oxford

Henry G.W., Hall D.S., 1991, ApJ 373, L9

Lacy C.H., Moffett T.J., Evans D.S., 1976, ApJS 30, 85

Micela G., Favata F., Pye J., Sciortino S., 1995, A\&A, in press

Minikulov N.Kh., 1987, Izv. Krym. Astrofiz. Obs. 76, 43

Pagano I., Ventura R., Rodonò M., Peres G., Micela G., 1995, A\&A, submitted 